\title{
La modelación como método del conocimiento científico en las ciencias sociales. El caso del modelo cubano de televisión local
}

Modeling as a method of scientific knowledge in the Social Sciences. The case of the Cuban local television model

\author{
Dagmar Herrera Barreda \\ Facultad de Comunicación. Universidad de La Habana, \\ Cuba \\ dagherrera@gmail.com \\ Hilda Saladrigas Medina \\ Facultad de Comunicación. Universidad de La Habana, \\ Cuba \\ saladrigas@fcom.uh.cu
}

\section{Resumen:}

El artículo sustenta la viabilidad del empleo de la modelación como método del conocimiento científico dentro de las ciencias sociales y, de modo particular, su aplicación al estudio de los procesos comunicativos mediáticos. Parte de definir la modelación, con sus ventajas y riesgos epistemológicos, para luego exponer los pasos seguidos en el análisis de la televisión de cobertura local en Cuba y la conformación de un modelo que posibilitó explicar el funcionamiento de este sistema así como sus potencialidades inexploradas para actuar en aras del desarrollo local. Se emplean como técnicas fundamentales para arribar al conocimiento, la investigación bibliográfica documental, la entrevista, la encuesta y la triangulación constante, siempre marcadas por las pautas de la modelación como método científico. Los resultados abren el camino para conformar una propuesta proyectiva de televisión para el desarrollo local en el contexto cubano.

Palabras CLAVE: Modelación teórica, Modelo televisivo, Televisión local, Televisión cubana, Televisión para el desarrollo.

\section{Abstract:}

The article supports the viability of the use of modeling as a method of scientific knowledge within the Social Sciences and, in particular, its application to the study of communicative media processes. It defines modeling with its epistemological advantages and risks, to then expose the steps followed in the analysis of local coverage television in Cuba and the creation of a model that makes it possible to explain the operation of this system as well as its unexplored potentials to act in for the local development. They are used as fundamental techniques to arrive at knowledge documentary bibliographic research, interview, survey and constant triangulation, always marked by the patterns of modeling as a scientific method. The results open the way for a projective television proposal for local development in the Cuban context.

KeYwords: Theoretical modeling, Television model, Local television, Cuban television, Television for development, Communication for social change.

\section{INTRODUCCIÓN}

La modelación o modelización es un método del conocimiento científico, "una praxis cognitiva que supone la construcción de una representación mental del objeto de la modelización” (Rodríguez y Roggero, 2014, p. 421).

Interpretando a Marvin L. Minsky (1965), puede decirse que la modelación es el proceso mediante el cual un observador (investigador) con intenciones (por qué y para qué) se aproxima a una realidad determinada que le interesa (concreta o conceptual) mediante prácticas indagatorias (empíricas y/o racionales) y elabora 
una representación de la misma (modelo) que expresa en un determinado lenguaje (natural/no formal o artificial/formal), el cual le ofrece respuestas que le interesan sobre dicha realidad.

Con amplia aplicación en las ciencias naturales y exactas, en las ciencias sociales su empleo implica riesgos epistemológicos (Rodríguez y Roggero, 2014). Pero se puede afirmar que

...constituye una estrategia para abordar la complejidad social (Morin, 1984; Roggero, 2006). Más aún, la modelización ofrece nuevas posibilidades para un trabajo colectivo de carácter verdaderamente interdisciplinario (García, 2006). Así, el trabajo de modelización y simulación brinda la posibilidad inédita de integrar conocimientos de distintas disciplinas y, por tanto, de abrir las ciencias sociales (Wallerstein, 1996) a un diálogo fecundo con las ciencias de la vida, las ciencias de la materia y las ciencias computacionales (Rodríguez y Roggero, 2014, p. 420).

Su utilización permite -como ya se ha mencionado- la obtención de un modelo del cual existen múltiples definiciones, a saber, "una estructura de símbolos y reglas operativas que proporcionan un conjunto de hechos relevantes de un proceso dado" (Detusch en Severiny Tankaed, 1991, p. 36); "cualquier sistema que se tome como representación de otro sistema" (García, 2002, p. 285); "representación de un dispositivo real mediante un sistema formal -por ejemplo un sistema de ecuaciones- que permite a la vez pensar este dispositivo, estudiar su funcionamiento y actuar sobre él para controlarlo o hacerlo evolucionar” (Armatte, 2006, p. 35); "la representación de aquellas características esenciales del objeto que se investiga, que cumple una función heurística, ya que permite describir y estudiar nuevas relaciones y cualidades de ese objeto de estudio con vistas a la transformación de la realidad" (Valle, 2007, p. 11); "un sistema intermedio auxiliar, material o ideal, resultado de un proceso creador de abstracción y generalización que con sus fundamentos teóricosprácticos, explica y representa con la lógica que caracteriza al objeto de estudio, las relaciones necesarias e imprescindibles" (Boullosa et al., 2009, p. 7).

A partir de estos referentes, la investigación que sirve de base al presente artículo inquirió sobre los elementos que caracterizan al sistema de la televisión de cobertura local en Cuba, con modos de actuación particulares y únicos -como objeto de referencia-, conformado por estructuras televisivas que, en las circunstancias sociohistóricas actuales, poseen potencialidades inexploradas de contribuir al desarrollo de los espacios municipales ${ }^{1}$ en que se ubican. Para ello el trabajo se sirvió de diversas técnicas de recogida de información como la investigación bibliográfica y documental, la entrevista, la encuesta y la triangulación constante. El resultado fue la obtención de un modelo actuante que se describe y explica, el cual desde el punto de vista comunicativo y según el comunicólogo español Manuel Martín Serrano, "tiene en cuenta sus componentes y las relaciones que existen entre ellos" (2005, p. 89).

A partir de las deficiencias detectadas mediante contrastación con las condiciones de posibilidad de desarrollo local de las estructuras sociales cubanas en el nivel municipal y con el apoyo de criterios de experticidad, se definen ejes fundamentales sobre los que podría proyectarse un modelo que representara los aspectos más significativos de una televisión local para el desarrollo de los municipios en el contexto cubano.

El artículo pretende demostrar, en primer lugar, que la modelación es un método científico factible de ser aplicado al estudio de los procesos comunicativos mediáticos y a su relación con las estructuras sociales que media -y por las que resulta mediado-, y, en segundo lugar, se busca demostrar que existe un modelo de televisión local en Cuba que permite explicar el funcionamiento de estos medios en los municipios del país. Este modelo resulta limitado en aras del cumplimiento de su encargo social en general, y, particularmente, en sus potencialidades para el actuar sistémico en aras del desarrollo local.

En tal sentido, están explicitados los argumentos y aparecen organizados los contenidos del artículo en su concepción metodológica, la presentación y discusión de los resultados, y las conclusiones. 


\section{Metodología}

\subsection{Elementos constitutivos de un modelo teórico para el estudio de objetos de referencia en las ciencias sociales}

Como método teórico, la modelación requiere de dos tipos de procesos o formas esenciales para su utilización: a) la aplicación en la práctica de un modelo ya establecido o conocido, y b) la obtención de un nuevo modelo (Boullosa et al., 2009, p. 3). El pedagogo cubano, Dr. Alberto del Valle Lima, por su parte, define que es posible establecer modelos y metamodelos; estos últimos como "modelos para obtener otros modelos, los cuales se conforman o estudian en un plano metateórico" (2007, p. 13) y no requieren ser definidos en todos sus componentes. A partir de la sistematización conceptual de estos presupuestos se construyó, en un primer momento, un metamodelo para el estudio de objetos de referencia en las ciencias sociales que

...constituye un modelo de modelos, es decir, un conjunto de elementos (conceptos, propiedades, relaciones) que permiten construir modelos. En términos más técnicos, un meta-modelo define la sintaxis del modelo (...) su objeto es la estructura formal para la construcción de modelos vinculados con cierto dominio de fenómenos (...) no tiene alcance universal, sino que tiene un ámbito de validez acotado al campo problemático en el que se inscribe el estudio (Rodríguez y Roggero, 2014, p. 429).

y se enuncian y diagraman (Figura 1) sus elementos constitutivos:

\section{FIGURA 1}

Diagramación de los elementos constitutivos de un modelo teórico

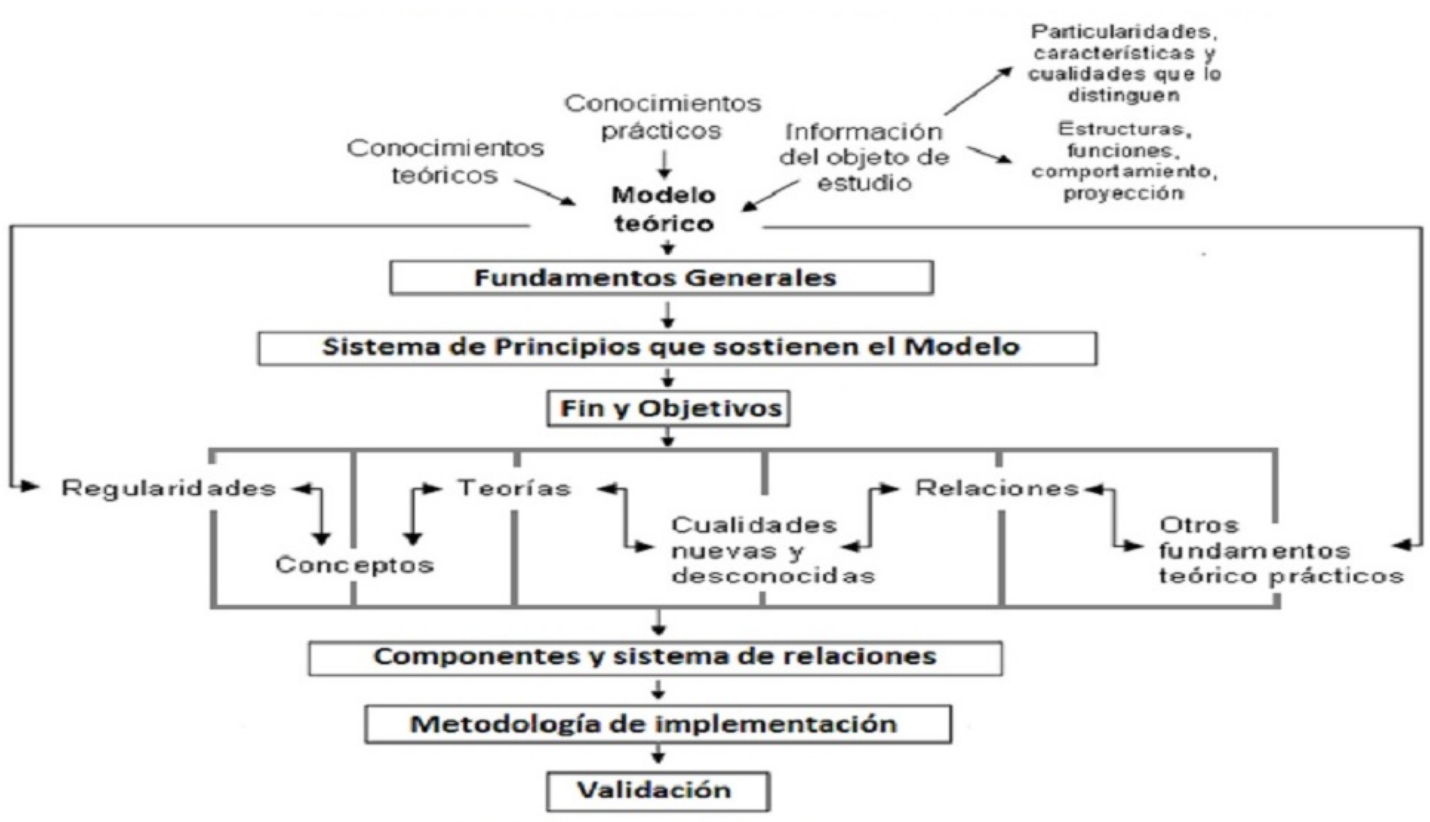

Fuente: Herrera (2015a)

- Presupuestos conceptuales: conjunto de teorías y conocimientos sobre los que se funda y sustenta el modelo.

- Referentes: conocimientos prácticos, referenciales, culturales, políticos.

- Información del objeto de estudio: particularidades, características y cualidades que distinguen a la realidad o sistema de referencia estudiado, su estructura, funciones, comportamiento y proyección. 
- Fundamentos: nociones fundacionales, esenciales, que dan origen y sostienen la propuesta, a partir de los presupuestos conceptuales y referenciales y de la información del objeto de referencia o estudio.

- Principios: proposiciones básicas, fundacionales y rectoras que guían la esencia y existencia del modelo.

- Fin: intención final que define la razón de ser del modelo.

- Objetivos: meta o metas a alcanzar con la realización del modelo. Aclara los porqués de la investigación.

- Variaciones y regularidades: atributos comunes y cambiantes de la realidad estudiada que permiten el análisis complejo y multifactorial.

- Conceptos: criterios establecidos y/o construidos para la obtención del nuevo conocimiento.

- Componentes: estructuras, ámbitos, actores y mediaciones que intervienen en el sistema analizado.

- Sistemas de relaciones: tejidos, vínculos, relaciones que se establecen entre los componentes y definen la esencia del sistema.

- Cualidades nuevas y desconocidas: características emergentes como parte de la indagación científica que permiten analizar y comprender al objeto desde nuevas y diversas perspectivas.

- Otros fundamentos teórico-prácticos emergentes en función de las características particulares del objeto interrogado y su relación con su sistema de referencia.

Se definen además tres elementos válidos, fundamentalmente, para los modelos proyectivos; o sea, aquellos que pretenden planear un futuro posible:

- Metodología de implementación: proyección a largo plazo que permite definir, planificar el rumbo a seguir para la obtención de los objetivos trazados.

- Procedimiento metodológico para su uso: definición de la metodología para la implementación de la estrategia que acota plazos, acciones, actores, objetivos.

- Validación: método de verificación que permite otorgar confiabilidad a los resultados alcanzados.

En un segundo momento de la investigación, a partir del metamodelo elaborado, se realizó el estudio de la televisión local en Cuba como objeto de referencia. El mismo dio como resultado un modelo actuante que representa los componentes, características y relaciones más significativas, las particularidades y regularidades reconocibles del estado actual de la televisión en el contexto local cubano, las cuales permiten su comprensión y abordaje conceptual. Se trata de una realidad, configurada a partir de la consolidación del trabajo de las organizaciones mediáticas locales, que hasta la fecha carece de estudios científicos que la tengan como referente.

El diseño, el reconocimiento de sus potencialidades y el diagnóstico de los modelos internacionales de referencia permitirán arribar, en un futuro inmediato, a una propuesta final de modelo proyectivo de televisión local para el desarrollo municipal. La misma constituye el estado ideal alcanzable de funcionamiento de la televisión local en el contexto cubano, ya que posibilitaría establecer modos de articulación/imbricación de las relaciones sociales que se producen en los espacios en los que actúa, con vistas a enfocarse -sin renunciar a su esencia- al desarrollo local.

El método de modelación fue aplicado en todo el recorrido investigativo desde visiones de ruptura y continuidad dialécticamente entrelazadas. Parte desde la práctica, la determinación y la sistematización de los rasgos del modelo actuante, y de las oportunidades emergentes en el escenario social, político y comunicativo nacional, no como fórmula lograda, sino como escenario deseado, perfectible y posible.

Esto fue posible, primero, por el empleo de varias técnicas para la recogida de información teórica y empírica, que constantemente fue sistematizada y ordenada por medio de la triangulación. A continuación se resume la ruta seguida, que contempla algunos de los elementos ya enunciados:

- Se sistematizaron las principales teorías, que sirven de fundamentos teórico-conceptuales al estudio; 
- Se realizó un diagnóstico de los modelos de televisión local de referencia en el ámbito internacional;

- Se levantó la información referida al objeto de estudio en el ámbito nacional, sus particularidades, características, cualidades, estructuras, funciones, comportamiento y proyección;

- Se construyó un modelo que permitió indagar en torno a los rasgos fundamentales y modos de actuación del sistema de televisión local cubano, carente hasta el momento de un pensamiento conceptual;

- Se indagó en las potencialidades atribuidas a la televisión local de contribuir al desarrollo local a partir de la teoría, el criterio de expertos, la visión de los actores de los procesos comunicativos audiovisuales, y los actores sociales del desarrollo local en el espacio municipal cubano;

- Se sometió el modelo resultante a validación a partir del criterio de expertos;

- Se reformuló la propuesta de modelo, objeto del ejercicio.

El recorrido investigativo se caracterizó por la flexibilidad y la posibilidad de incorporar los datos que fueron emergiendo, en una construcción cíclica y ascendente del conocimiento que le confiere solidez a los resultados obtenidos.

Para potenciar la visión holística de los estudios cualitativos (Taylor y Bogdan en Rodríguez et al, 1996, p. 33), como método complementario se emplearon elementos de la Matriz DAFO para el diagnóstico nacional, a partir del análisis interno y externo del entorno, con el objetivo de sortear las amenazas, aprovechar las oportunidades, reducir el impacto de las debilidades y lograr los objetivos sostenidos en las fortalezas.

Además, fueron de vital utilidad técnicas como:

- la investigación bibliográfica y documental, para el acercamiento a las teorías y referentes que sustentan la investigación a partir de la consulta de fuentes documentales primarias y secundarias;

- el análisis de contenido cualitativo a documentos normativos y parrillas de programación de los canales cubanos de cobertura local, para determinar, fundamentalmente a nivel temático, la presencia de términos, conceptos o concepciones presentes o subyacentes y sus relaciones con los contextos de producción y reproducción;

- la entrevista, efectuada a directivos, expertos y especialistas nacionales e internacionales, y trabajadores de canales locales de televisión, así como a actores locales, en sus modalidades en profundidad, semiestrandarizada y estandarizada (Alonso y Saladrigas, 2002), a un total de 158 sujetos;

- la encuesta, aplicada a 139 trabajadores de canales y corresponsalías municipales -vinculados fundamentalmente con la producción informativa-, que permitió obtener datos puntuales, cuantitativos y cualitativos, del funcionamiento de la televisión local en Cuba, las características de su oferta comunicativa y los rasgos mantenidos en el tiempo, así como posibles vínculos con el desarrollo de los espacios en que accionan estas entidades mediáticas.

La mayoría de estas técnicas fueron aplicadas de 2008 a 2015, año de cierre de la investigación. Como constante, la triangulación (Cisterna, 2005, p. 68) permitió lograr la reunión y cruce dialéctico de toda la información pertinente al objeto de estudio surgida en la investigación empírica y teórica, que, en esencia, constituye el corpusde resultados de la investigación. 


\section{Resultados y Discusión}

\subsection{Fundamentos teórico-conceptuales del modelo de televisión local para el desarrollo municipal: presupuestos conceptuales de partida}

De forma particular, en la investigación comunicológica los modelos cumplen una función organizadora; ordenan y relacionan los elementos entre sí para dar una imagen completa de los procesos que ayudan a explicar de manera simplificada. Sirven de guía a los investigadores para dirigir sus estudios hacia partes concretas del proceso de la comunicación y posibilitan el pronóstico de los acontecimientos (McQuail y Windahl, 1997, pp. 30-31).

A los efectos investigativos, los modelos construidos para explicar la televisión se han estudiado frecuentemente desde aspectos como los objetivos de la programación, los horarios, la presencia o no de anunciantes y el tiempo que se les asigna a éstos. Otros tópicos valorados suelen ser el origen y destino de la señal, su titularidad, el cubrimiento territorial, la orientación de la programación, los usuarios y la tecnología de transmisión. Algunos autores (Gumucio-Dagron, 2002, 2006; Cebrián, 2003) los segmentan para su análisis en estatales, comerciales y comunitarios, en correspondencia con los sectores privado, público y comunitario de la comunicación.

Según la orientación general de la programación, el teórico boliviano Alfonso Gumunio-Dagron (2002) determina los modelos actuantes de la televisión comercial y la televisión de interés público, social, educativo y cultural. El primero, sin excluir necesariamente los propósitos educativos, recreativos y culturales, tiene como elemento distintivo fundamental el ánimo de lucro, a través de una programación destinada a la satisfacción de los hábitos y gustos de los televidentes (Angulo y Zabaleta, 2008, p. 2). La televisión de interés público, por su parte, suele ser desarrollada por los Estados para satisfacer necesidades educativas y culturales, y, por lo general, suele comercializarse y/o recibir subvenciones públicas para lograr su sustentabilidad económica.

En el caso particular que nos ocupa, y en dependencia de sus objetivos, vocación y finalidad, la televisión local puede ser tanto comunitaria, pública/estatal, como privada/comercial.

Dentro del campo de la comunicación, y como resultado de una búsqueda inicial para la conformación de la propuesta de este artículo, se ha podido identificar una carencia de estudios sustentados en la modelación como método del conocimiento científico. Esto se puede justificar a partir del empleo indistinto de los conceptos modelo y modelación, y el uso del término modelo para aludir al objeto de referencia y no a su representación abstracta, la cual, en el mejor de los casos, refiere a la representación gráfica de los procesos comunicativos. Es por ello que, en el presente artículo, se proponen transferencias de modelos de otros tipos de procesos - pedagógicos y económicos fundamentalmente- hacia los comunicativos, y se asume el método -resumiendo las definiciones ya enunciadas- como la configuración ideal que representa una teoría de manera simplificada. Dicha teoría es resultado de un recorrido creador de abstracción y generalización que, con sus fundamentos teóricos-prácticos, representa las relaciones necesarias e imprescindibles mediante conceptos, propiedades, regularidades, cualidades, signos y sus relaciones, cuya función básica es la de ayudar a comprender las teorías y las leyes mediante la representación de aquellas características esenciales del objeto que se investiga, el cual cumple una función heurística, ya que permite describir y estudiar nuevas relaciones y cualidades con vistas a la transformación de la realidad.

Como núcleos teóricos se abordan la sociedad global (las principales características de la actual sociedad global-local), lo local como espacio de acción y cómo se inserta en ella la televisión. Se ofrecen los elementos esenciales que caracterizan a los sistemas de comunicación pública en el espacio local, se define el desarrollo, el desarrollo local, y las concepciones en torno a él, con énfasis en su perspectiva sociocultural, en particular desde el enfoque de la multiplicidad. Se resumen los principales elementos del debate referente a la comunicación para el desarrollo y el cambio social, como marco de actuación de los medios locales, y se realiza 
una sistematización de los principales modelos internacionales de televisión local (modelos de referencia) y sus relaciones con el desarrollo local (Norteamérica: Canadá y Estados Unidos; Latinoamérica: Argentina, Brasil, Colombia y Venezuela, y Europa: Francia, España, Bélgica y Holanda) junto con estudios referidos a la televisión local desde Latinoamérica y Europa como resultado.

Los modelos analizados, si bien difieren en sus prácticas y en su mayoría no proponen un cambio social desde los medios, brindan elementos a considerar en el intento por establecer procesos comunicativos ciudadanos en los que la televisión local se convierta en el centro del desarrollo.

En función del recorrido realizado, y como parte de sistematizaciones ofrecidas por las autoras (Herrera y Saladrigas, 2017, p. 207), se determinan como puntos de partida los siguientes:

- En el contexto actual, la comunicación es una potente herramienta de cambio social, desarrollo y participación ciudadana, por tanto, definir el tipo de sociedad que queremos presupone también definir el tipo de comunicación que debe primar en esa sociedad.

- Es imprescindible la comprensión y el análisis de la televisión y los medios en el espacio local con relación a los sistemas comunicativo y social en los cuales se insertan y de los que son parte integrante. El intercambio con estos sistemas va a conferir las características particulares a cada medio que, en el caso local, adquiere singularidades por la constante interacción entre los ámbitos micro y macro.

- Desde la televisión que se realiza en los espacios locales es posible generar propuestas transformadoras que serán más efectivas e incidirán más en el espacio en el que se implementen, en tanto mayores sean los niveles de participación, la apropiación de la ciudadanía con el medio comunicativo y su posibilidad de decisión sobre los cómo, cuándo, por qué, para qué y con quiénes de esa televisión.

- Desde la televisión en el espacio local, una verdadera propuesta de cambio social debe apostar por asumir el desarrollo local, más que como un proceso endógeno de cambio estructural, como el "empoderamiento" de una sociedad local (Boisier, 2005, p. 6), lo cual es posible a partir del enfoque de la multiplicidad o del otro desarrollo y del modelo participativo u orgánico de la comunicación.

- Un modelo de televisión local para el desarrollo municipal debe ofrecer igualdad de oportunidades para todos los miembros de la localidad, garantizar la sostenibilidad de la propuesta y la potenciación de la vertiente humana del desarrollo, de manera que los actores sociales locales puedan participar y beneficiarse de los proyectos que gestan e implementan.

- Una propuesta transformadora de la televisión local debería rebasar el rol de inductora e indicadora de cambio social que se le concedía a la comunicación a mediados del pasado siglo como parte de los enfoques modernizadores y de dependencia persistentes a partir de la voluntad de promover ese desarrollo, con una vocación comunitaria, con incentivo a la participación ciudadana, desde el pleno conocimiento de los recursos y necesidades locales; un abordaje endógeno, sostenible y en constante interacción con el sistema de referencia.

Se define a la televisión local para el cambio social como un fenómeno de relación sociocultural y crecimiento colectivo, fortalecedor del sentir comunitario, con bases en el enfoque de la multiplicidad y vínculo directo con proyectos o estrategias de desarrollo, que sea un actor más del desarrollo local y actúe de conjunto con el resto de los actores; que ejecute acciones, contribuya a fijar y dé a conocer el rumbo que ha decidido tomar el municipio, a partir de garantizar contenidos locales e información de calidad como un derecho vital para la ampliación de oportunidades de los seres humanos; que viabilice el acceso ciudadano al medio y la apropiación de sus procesos comunicacionales mediante la participación sustantiva, el diálogo horizontal, el intercambio participativo el debate y la negociación; que fortalezca la capacidad expresiva de la gente, facilite los enlaces e intercambios entre individuos y agrupaciones para robustecer el tejido social en su integridad, donde más que la información transmitida prime el significado creado, compartido y generado de conjunto y desarrolle una vocación de servicio público acorde con los valores y normas de cada comunidad y los principios de la sociedad socialista cubana. 
Con estos fundamentos, que constituyen los presupuestos conceptuales de partida, se procede a la determinación de los conocimientos prácticos y las características del objeto de estudio que permiten establecer los rasgos distintivos del modelo actuante de televisión local en Cuba. Luego, se define la concepción nacional sobre desarrollo local, las características del contexto actual que propician o dificultan la implementación de la propuesta, y se concluye con un diagnóstico de las debilidades, fortalezas, amenazas y oportunidades del modelo actuante.

\subsection{Referentes del modelo de televisión local para el desarrollo municipal en el contexto cubano}

La existencia de canales de televisión en el espacio municipal cubano destaca como un elemento significativo y de trascendencia dentro del sistema de televisión nacional, y es una muestra evidente de su evolución. Surgen de forma gradual, a partir de 2004, con la premisa de vincular las facilidades tecnológicas municipales -tanto de instituciones como privadas-, para producir material audiovisual local e informar a la población ante situaciones excepcionales.

El accionar de estas estructuras mediáticas, la homogeneidad en sus bases estructurales e infraestructurales los llevan a integrar un subsistema de televisión de cobertura local, integrante a su vez del Sistema de la Televisión Nacional y del Sistema de Comunicación Nacional y a definir, en el hacer cotidiano y sin proponérselo, modos de actuación, propios, autónomos y auténticos, que han sido estudiados como parte de una línea de investigación dentro del campo de la comunicación en Cuba.

Sin embargo, las potencialidades inexploradas de esa televisión, puestas de manifiesto en estudios de pregrado y postgrado, la presencia de un modelo estatal de prensa que debe insertarse en el proceso de perfeccionamiento del modelo social cubano y las características de una sociedad que de forma ascendente se interesa en impulsar el desarrollo local -a partir de un diseño propio centrado en el fortalecimiento de las potencialidades municipales para nivelar las condiciones socioeconómicas de las distintas regiones del paíshan dado como resultado un comportamiento que provoca que la televisión local cubana desaproveche sus posibilidades de contribuir al desarrollo municipal. Esta situación conduce a centrar la mirada en la forma sin proposiciones previas en el contexto nacional - en la cual esta televisión puede contribuir al desarrollo local de los municipios cubanos, máxime cuando priman concepciones no comunicativas en torno al desarrollo.

Es por ello que, como parte del estudio "Televisión local y desarrollo: Articulaciones desde lo Local”, que constituyó la investigación para optar por el grado de doctor en Ciencias de la Comunicación (Herrera, 2015a), se propone y fundamenta el modelo actuante de televisión local en Cuba, como paso para obtener una propuesta proyectiva del modo en que el medio, al colocar la participación ciudadana como eje transversal dinamizador, puede tributar al desarrollo municipal en el contexto cubano.

Como caso particular, la televisión local en Cuba surge por la necesidad de ofrecer una alternativa televisiva a las provincias orientales a las que solo llegaba la señal de un canal nacional y con el paso de los años se fue articulando un sistema estructurado, con características, lineamientos, misiones propias y distintas a las de la Televisión Nacional, que aumenta la visibilidad y representatividad de los diferentes sectores e intereses territoriales en el medio, tanto en la provincia como a nivel nacional. Como resultado descentraliza y complementa al Sistema Nacional de Televisión, dado que llena los vacíos comunicacionales existentes mediante el empleo de los recursos técnicos y el personal de cada territorio.

\subsection{Rasgos fundamentales del modelo actuante de televisión local en Cuba2}

Se parte de establecer que, en el caso cubano, las características del sistema social y la estructura políticoadministrativa de la Isla definen, necesariamente, cualquier clasificación de sus medios de comunicación. De 
esta forma, y en correspondencia con las regiones en que se divide el país, es posible determinar televisiones a escala nacional, provincial y municipal o local, en las que el ámbito de difusión y cobertura cobra trascendental importancia. $^{3}$

A los efectos investigativos, pueden identificarse cinco tipologías ${ }^{4}$ fundamentales de televisión en el espacio regional y local cubano 5 (Herrera, 2015b):

- Canales provinciales,

- Centros de producción audiovisual con transmisiones provinciales a través de un canal nacional (Telecentros), ${ }^{6}$

- Canales municipales de centros provinciales (Canales municipales de telecentros provinciales),

- Canales municipales de televisión con transmisiones locales y

- Corresponsalías municipales de televisión con transmisiones locales.

Se reconoce a las últimas tres tipologías como los componentes representativos de la televisión de cobertura local en Cuba, que se pueden comprender a partir de la conformación de un modelo teórico no formal.

En relación con sus principios, el modelo de televisión local cubana se distingue por ser estatal, de titularidad pública, con un marcado carácter informativo cultural, sin fines comerciales, que pertenece al Instituto Cubano de Radio y Televisión (ICRT), a su sistema de televisión nacional, y posee vínculos estrechos con el Partido Comunista de Cuba (PCC) y el Gobierno en sus escalas nacional y local. Actúan según el precepto de medios del pueblo y para el pueblo.

En correspondencia, sus objetivos fundamentales son: realizar una programación informativa variada, que refleje el acontecer económico, político y social del territorio; divulgar la constante superación educacional, histórica, científico técnica, artística y cultural; intervenir en la creación de hábitos y gustos; cumplir con las orientaciones establecidas por el partido provincial y municipal con respecto a la política informativa, propaganda, educación, arte y cultura y con lo establecido en la política de programación de la TVC, y ser un instrumento de orientación revolucionaria y político-ideológica del Municipio. Se concibe como medio en el que se vea reflejada la localidad.

Es dirigido a nivel nacional desde la presidencia de televisión del ICRT y a nivel provincial desde la figura del telecentro provincial. Su estructura se expresa en los componentes locales de los sistemas televisivos territoriales o los de radio y televisión en el caso de la variación experimental, y conforma el sistema de televisión de cobertura local cubano. Se compone de dos variaciones: una tradicional y otra experimental sin que por ello pierda su identidad y esencia- (Figura 2).

FIGURA 2

Variaciones de los sistemas de televisión local en Cuba
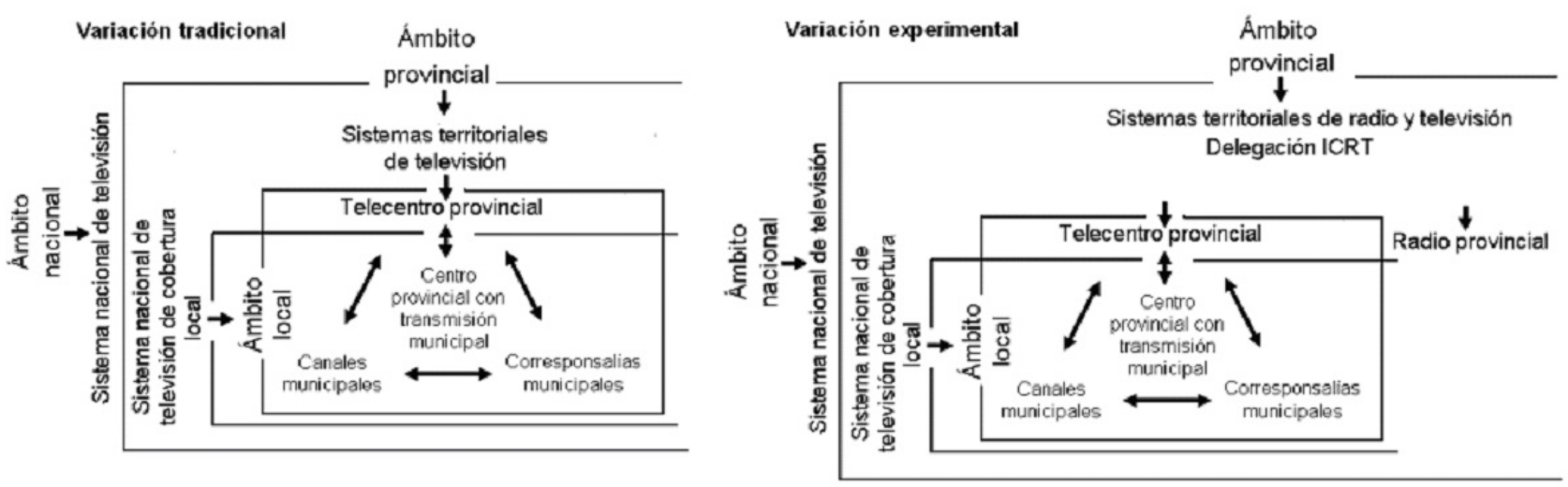

Fuente: Herrera (2015a) 
La primera se corresponde con la práctica consolidada de los sistemas territoriales de televisión, a partir de que en 2006 se completara la implementación de las estructuras locales. En este sistema el telecentro funciona como la dirección de televisión de su territorio y, como tal, dirige y centra el trabajo de los canales y las corresponsalías municipales. Además, existe una variación experimental -puesta en práctica a partir de 2011 en las provincias Artemisa y Mayabeque-, en la que, como parte de las nuevas reestructuraciones político-administrativas, se introduce la figura de la Delegación del ICRT, que orienta y organiza el trabajo del sistema de radio y televisión en cada uno de estos territorios, sin que el telecentro provincial pierda su rol de coordinador de las estructuras televisivas, ahora más enfocado en la programación. Esta experiencia se espera implementarla a nivel nacional.

Las relaciones entre sus componentes -canales municipales de los telecentros provinciales, canales municipales y corresponsalías municipales con transmisiones locales-varía en función de las particularidades de cada territorio, aunque por lo general no existen articulaciones directas entre ellas, están mediadas por la figura del telecentro provincial, que rige el trabajo audiovisual, y al que tributan de forma sistemática y planificada con sus productos comunicativos, los cuales luego se destinan a la televisión nacional. La televisión local actúa de forma más consolidada en las experiencias de los canales municipales y corresponsalías municipales con transmisiones locales y debe aún ganar en autonomía a nivel de propuestas de programación en el caso de los canales de los telecentros provinciales.

Las televisoras de cobertura local se han insertado como canales que producen y transmiten programaciones para sus municipios, a la vez que han desempeñado el rol de corresponsalías que hacen llegar a la provincia o a la nación lo más relevante del acontecer local, garantizando la presencia de ese acontecer en mayores ámbitos de difusión. Su oferta comunicativa se caracteriza por ser de factura nacional, en gran medida propia -a excepción de algunos canales de telecentros provinciales-. En ella se refleja la localidad y sus temas de interés. En correspondencia, las fuentes locales son las más empleadas. Los tópicos provinciales o nacionales ocupan un espacio reducido en sus ofertas y la agenda del medio se conforma fundamentalmente a partir de las propuestas de la dirección del canal y, en mucha menor medida, por sugerencias del público o de las instituciones locales, hecho que refuerza la estructura vertical del modelo actuante.

La participación de la comunidad por lo general se evidencia en los mensajes (Kronhling 2004), a partir de su presencia como principal asunto de significación/ sentido (Gutiérrez, 1998), o como tema principal de la programación. Existen canales y, más puntualmente, programas con vocación comunitaria en los que se establece una comunicación horizontal, dialógica y participativa. No obstante, prima el apego a los referentes estéticos y formales nacionales y a la reproducción de esquemas transmisivos unilaterales. Carece de vías efectivas para retroalimentarse con el público y medir así la efectividad de sus propuestas comunicativas.

Hasta la fecha de cierre de la investigación estaba integrado por 53 componentes: 10 canales municipales, resultado de la extensión de los TCP (18,86\%), 15 canales municipales con transmisiones locales $(28,30 \%)$ y 28 corresponsalías de televisión con transmisiones locales (52,83\%). Estas cantidades pueden fluctuar en la medida en que se abran, cierren o reformulen las funciones de cada una de las estructuras locales, sin que se afecten los principios del modelo ni éste en sí mismo.

El espacio local en la experiencia cubana está determinado por el área de cobertura de los transmisores, con presencia directa en algo más del $47 \%$ de los municipios cubanos. Éste, sin embargo, es actualmente un tema en constante cambio debido a la migración digital.

Más allá de que se ubica en los espacios locales, se nutre de los referentes latinoamericanos y posee algunas iniciativas puntuales que se vinculan estrechamente con el público, resulta poco adecuado denominarla como una televisión comunitaria. Sin embargo, posee indudables potencialidades para convertirse en una televisión de proximidad -en una fusión de sus variantes ventana y espejo (Musso, 1991) - en la que el público local se pueda ver reflejado en su actuar cotidiano, a la vez que encuentre espacios para asomarse y ver lo que sucede en otros lugares del país, del mundo. 
La televisión local, como parte del STVC y dentro de la concepción de la sociedad socialista, opera según consta en sus principios rectores- en correspondencia con las exigencias del desarrollo integral de la economía y de la sociedad. Dentro de él es posible introducir cambios que contribuyan a potenciar, con el empleo de herramientas comunicativas, el desarrollo local de los municipios en los que se inserta. En estos espacios presentan innegables aportaciones, que cualificamos como positivas y relevantes, y que constituyen sus principales fortalezas; sin embargo, son aún limitadas e insuficientes, puesto que falta por consolidar una gestión estratégica participativa de base local, con actores conscientes de sus potencialidades y posibilidades de transformación. Las potencialidades de la televisión local de incidir en el desarrollo municipal, refrendadas en la teoría y las referencias analizadas, superan con creces a la práctica actual.

El diseño particular del desarrollo local asumido en Cuba, sus fundamentos, la identificación de sus potencialidades y obstáculos constituyen condiciones favorables de partida para la generación de cambio social a través de la comunicación y de la televisión en particular, en un contexto de reestructuraciones refrendado en los Lineamientos de la Política Económica y Social del Partido y la Revolución Cubana (2011), que potencia la articulación de la mayoría de los procesos sociales, políticos y económicos desde el espacio local.

\subsection{Potencialidades de la televisión local de incidir en el desarrollo municipal en el contexto cubano}

Las tipologías de televisión definidas en el espacio regional cubano garantizan la presencia de estructuras televisivas en cada una de las provincias del país - y el municipio especial, Isla de la Juventud- y la presencia del medio en un total de 80 de los 168 municipios.

Según lo anterior, cerca de la mitad del territorio nacional, con su población y las informaciones que a ella le conciernen, debería encontrar presencia al menos una vez a la semana en el medio audiovisual. A este dato es necesario añadir que la mayoría de los municipios con estructuras audiovisuales figuran entre los de mayor concentración poblacional e importancia social, económica y político-administrativa dentro de la Isla. Además, un gran número de canales y corresponsalías municipales atienden, desde el punto de vista noticioso, a los municipios colindantes o zonas de interés dentro del territorio, que carecen de estructuras televisivas propias. Esto aumenta en un nivel significativo los porcentajes mencionados. Si bien existe una parte importante del territorio sin cubrir, la actual situación se muestra como muy superior en comparación con otros contextos internacionales y otras épocas nacionales. Se demuestra que existe en Cuba una infraestructura televisiva amplia, extendida a lo largo del territorio nacional y con importante presencia en los municipios que, en sintonía con las circunstancias sociohistóricas y políticas descritas, coloca al medio audiovisual en una coyuntura favorable, en la que es susceptible de ser empleado de forma consciente e intencionada, para lograr mejorías en los espacios en que se inserta, trascendiendo su mero rol transmisivo para convertirse en una televisión para el desarrollo local en correspondencia con los preceptos de la comunicación para el cambio social.

Es por ello que, una vez obtenidos los resultados a través de la modelación, se decidió determinar las potencialidades de la televisión local de incidir en el desarrollo municipal, a partir de los criterios de los actores de la televisión local en Cuba, que laboran en los canales y corresponsalías municipales, o que se vinculan directamente a ellas: actores del desarrollo local, materializados en representantes municipales del Gobierno y el PCC, expertos nacionales e internacionales y la teoría. ${ }^{7}$

La triangulación de la información proveniente de estas fuentes evidencia que la televisión local para el desarrollo municipal debe partir de la voluntad de promover ese desarrollo, con una vocación comunitaria, con incentivo a la participación ciudadana desde el pleno conocimiento de los recursos y necesidades locales; un abordaje endógeno, sostenible y en constante interacción con el sistema de referencia. De igual modo es 
posible enunciar ejes fundamentales sobre los que debe basarse una televisión local para el desarrollo de los municipios:

- Función informativa: Producir una mirada cercana inmediata al entorno próximo. Facilitar la comprensión de lo que ocurre en el entorno a partir del conocimiento, el compromiso, la información de calidad.

- Función informativa - analítica - reflexiva - problematizadora: análisis de la realidad local. Presentación y seguimiento hasta la posible solución de problemáticas locales.

- Participación ciudadana: como proceso sustantivo vital con bases en el diálogo y vía para desarrollar la democracia, a partir de su potenciación y de un modelo comunicativo horizontal.

- Vinculo con actores e instituciones locales: como representantes territoriales claves para el desarrollo, de los que es parte y con los que debe mantener una estrecha relación.

- Correspondencia con objetivos del desarrollo local: necesaria articulación y correspondencia entre la planificación estratégica del medio y los objetivos de desarrollo de su espacio de acción.

- Canalización y expresión: espacio, foro, plataforma permanente de presencia y debate para los colectivos y grupos sociales, otorgando un espacio reservado a las asociaciones minoritarias. Democratización de la vida pública y fomento de la pluralidad.

- Dinamización cultural, social y económico: desde los procesos que propicia, genera, incentiva y acompaña.

- Configuración del espacio local: a partir de la cohesión, construcción identitaria, vertebración del territorio, corresponsabilidad y autogestión. Reforzamiento de la realidad local.

- Identidad local: el desarrollo, afianzamiento, reforzamiento y/o creación de la identidad localcomunitaria de los colectivos sociales.

- Valorización individual y colectiva: contribuye a la toma de conciencia y generación de actitudes responsables al ser reflejados en pantalla los actores locales.

- Gestión descentralizada: que le confiera dinamismo, autonomía y le posibilite incidir de forma efectiva en el desarrollo local.

- Interacción constante con el ámbito global: colaboración entre canales. Complemento a la televisión nacional y regional.

La determinación del modelo de televisión local en Cuba y de sus potencialidades para incidir en el desarrollo local y el análisis de los modelos internacionales de referencia marcan las pautas para la posterior definición de una propuesta de modelo de televisión local para el desarrollo municipal en el contexto cubano.

\section{Conclusiones}

- El recorrido investigativo seguido y los resultados arrojados demuestran la validez de la modelación como método científico, el cual permite el estudio de los procesos comunicativos mediáticos y su relación con las estructuras sociales. De igual modo, se manifiesta la aplicabilidad de los elementos constitutivos del modelo teórico propuesto para el estudio de objetos de referencia en las ciencias sociales, toda vez que sirvió de base para el análisis del sistema de televisión local en Cuba -como parte de los sistemas de televisión territorial y nacional-, a partir de la conformación de un modelo de televisión local como representación abstracta y simplificada de la realidad empírica.

- El modelo resultante, en su carácter de representación teórico formal basada en supuestos científicos e ideológicos y a partir de su función sustitutiva-heurística, se constituye en una representación comunicativa de la realidad a partir de los elementos que la integran.

- La construcción del modelo actuante, sumada a la identificación de las potencialidades de la televisión de cobertura local de incidir en el desarrollo local y en el establecimiento de líneas de 
ruptura/ continuidad, permite comprender al objeto de referencia y determinar sus potencialidades inexploradas, lo que tributa, en una nueva fase, a la conformación de una propuesta alcanzable de televisión local que contribuya al desarrollo municipal en el contexto cubano.

\section{Bibliografía}

Angulo, L., y Zabaleta, U. I. (2008). Cinco estrategias para un modelo de televisión comunitaria: una perspectiva desde la experiencia colombiana. Recuperado de: http://www. aeic. org/malaga2010/upload/ok/183. pdf

Alonso, M. M. \& Saladrigas, H. (2002). Para investigar en comunicación social. Guia Didáctica. La Habana: Editorial Pablo de la Torriente.

Armatte, M. (2006). La Noción de Modelo en las Ciencias Sociales. EMPIRIA. Revista de Metodología de Ciencias Sociales (11) 33-70

Boullosa, A., Lage Jiménez, J. C. y Hernández Menéndez, E. E. (2009). La modelación y los modelos teóricos en la ciencia. Una concreción en la auditoria interna con enfoque de riesgo. Contribuciones a la Economía, julio. Recuperado de http://www.eumed.net/ce/2009b/tjm.htm

Cebrián, M. (2003). Enseñanza virtual para la innovación universitaria. Madrid: Narcea Ediciones.

Cisterna, F. (2005). Categorización y triangulación como procesos de validación del conocimiento en investigación cualitativa. Revista Teoría, 14, 61-71.

García, M. (2007). El desarrollo local. En M. García Rabelo (comp.) Las teorias acerca del subdesarrollo: una visión crítica (pp. 65 - 79). La Habana: Editorial Félix Varela

García, R. (2006). Sistemas complejos. Conceptos, método y fundamentación epistemológica de la investigación interdisciplinaria. Barcelona: Gedisa

Gumucio-Dagron, A. (septiembre, 2002). Comunicación para el cambio social. Clave del desarrollo participativo. Ponencia presentada en XX Encuentro Académico de la Asociación Colombiana de Facultades de Comunicación Social-AFACOM, Medellín, Colombia.

Gumucio-Dagron, A. (2006). Arte de equilibristas: la sostenibilidad de los medios de comunicación comunitarios. Recuperado de www.comunica.org/onda_rural/docs/gumucio.doc

Gumucio-Dagron, A. (2011). Comunicación para el cambio social: clave del desarrollo participativo. Signo y Pensamiento, 58 (XXX), 26-39.

Gutiérrez, M. (1998). La democracia en la Media. Recuperado de http://commpositEstados /Unidosqam.ca/videaz/ docs/magues.html

Herrera. D. \& Saladrigas H. (2017). Televisión local y cambio social: una propuesta de modelo participativo para el desarrollo municipal desde el contexto cubano. Revista Latinoamericana de Ciencias de la Comunicación XIV (26) 204-213.

Herrera. D. (2015a). Televisión y Desarrollo. Articulaciones desde lo local. Propuesta de modelo de televisión local para el desarrollo de los municipios en el contexto cubano (Tesis Doctoral inédita). Facultad de Comunicación, Universidad de La Habana, Cuba.

Herrera, D. (2015b). La televisión local en Cuba. Un sistema en desarrollo. Revista Razón y Palabra. Ecología de los medios, 89. Recuperado de http://www.razonypalabra.org.mx/N/N89/V89/10_Herrera_V89.pdf

Herrera, D., Saladrigas. H. \& Garcés. R. (2015c). Modelo de televisión local en Cuba. Una aproximación pionera a sus rasgos distintivos. Razón y Palabra, Comunicologia Cubana, (92). Recuperado de http://www.razonypalabra.or g.mx/N/N92/Monotematico/19_HerreraSaladrigasGarces_M92

Krohling, C. M. (2004). Televisión Comunitaria en Brasil: Antecedentes y participación popular en la gestión y en la programación. Revista Redes.com, 3, 347-367.

McQuail, D. y Windahl, S. (1997). Modelos para el estudio de la comunicación colectiva. Pamplona: Universidad de Navarra. 
Minsky, M. (1965). Matter, Mind and Models. Recuperado de https://groups.csail.mit.edu/medg/people/doyle/gall ery/minsky/mmm.html

Musso, P. (1991). La télévision dans les régions d'Europe. Lille: Editions Moroirs.

Rodríguez, G., Gil, J. y García, E. (1996). Metodología de la investigación cualitativa. Málaga: Editorial Aljibe.

Rodríguez, L \& Roggero, P. (2014). La modelización y simulación computacional como metodología de investigación social. Polis Revista Latinoamericana, 13 (39). Recuperado de http://dx.doi.org/10.4067/S0718-6568201400 0300019

Roggero, P. (2006). De la complexité en sociologie: évolutions théoriques, développements méthodologiques et épreuves empiriques d'un projet sociologique. Mémoire d'habilitation à diriger des recherches en sociologie. Toulouse: Université de Toulouse.

Serrano, M. (2005). Teoría de la Comunicación. Epistemología y Análisis de Referencia. La Habana: Editorial Pablo de la Torriente.

Severin, W. y Tankaed, J.W. (1991). Communication theories: origins, methods and uses in the mass media. New York: Longman

Valle Lima, A. (2007). Metamodelos de la investigación pedagógica. La Habana: Ministerio de Educación. Instituto Central de Ciencias Pedagógicas.

Wallerstein, I. (1996). Abrir las ciencias sociales. Informe de la Comisión Gulbenkian para la reestructuración de las ciencias sociales. México: Siglo XXI.

\section{Notas}

1 Se corresponde con el espacio geográfico mínimo de la división política administrativa cubana con expresión jurídica.

2 El presente epígrafe sintetiza algunos resultados publicados con anterioridad en la Revista Razón y Palabra (Herrera, 2015; Herrera, Saladrigas y Garcés, 2015)

3 El Sistema de la Televisión Cubana (STVC) está compuesto por: un canal internacional: Cubavisión Internacional, que se corresponde con la televisión transnacional;cinco canales nacionales: Cubavisión, Tele Rebelde, Canal Educativo, Canal Educativo 2 y Multivisión, que se corresponden con la televisión nacional; un sistema de televisión territorial que se corresponde con la televisión territorial o regional y está conformado por: 15 telecentros provinciales, un canal provincial (Canal Habana), una productora de audiovisuales comunitarios (Televisión Serrana); un sistema de televisión de cobertura local, inscrita en de las televisiones locales/municipales e integrada por: canales y corresponsalías municipales.

4 En tanto constituyen una representación simplificada de la realidad que puede ser contrastada de forma empírica.

5 Bajo estas mismas condiciones (pertenecer al ICRT y ser de servicio público), podría definirse como una sexta tipología a Televisión Serrana, sin embargo, no es en sí misma un canal, sino una productora de audiovisuales comunitarios sin homologías en el contexto nacional, por lo que preferimos mantenerla como un caso aparte y singular.

6 Reconocidos junto a la denominación anterior como la televisión provincial de Cuba.

7 Sus valoraciones fueron recogidas mediante entrevistas en profundidad (a 8 directivos de la Televisión Cubana y 5 expertos nacionales e internacionales), semiestandarizadas (a 36 directivos y trabajadores de la televisión local cubana) y estandandarizadas (mediante cuestionario a un total de 124 trabajadores de canales locales, especialistas nacionales, expertos internacionales y actores del desarrollo local. Entre ellos, a 105 dirigentes políticos y gubernamentales de 57 municipios con estructuras televisivas). 